\title{
Bizarre presentation of bilateral ovarian leiomyoma: a case report
}

\author{
VICTORIA C. LEMA ${ }^{1,2}$, ANTHONY N. MASSINDE ${ }^{1,2^{*}}$, PETER F. RAMBAU ${ }^{3}$, EDGAR \\ NDABOINE ${ }^{1,2}$, DISMAS MATOVERO ${ }^{2}$ and RICHARD NY. RUMANYIKA ${ }^{1,2}$ \\ ${ }^{1}$ Department of Obstetrics \& Gynaecology, Bugando Medical Centre, P.O. Box 1370, Mwanza, \\ Tanzania \\ ${ }^{2}$ Department of Obstetrics \& Gynaecology, Catholic University of Health Sciences and allied \\ sciences, P.O. Box 1464, Mwanza, Tanzania \\ ${ }^{3}$ Department of Pathology, Catholic University of Health Sciences and allied Sciences, P.O. \\ Box 1464, Mwanza, Tanzania.
}

Abstract: Primary leiomyoma of the ovary is rarely described in literature. It accounts for $0.5-1 \%$ of all benign ovarian tumours. Bilateral ovarian leiomyoma are rare and only few cases have been described. In this report, we describe a case of 29-year-old nulliparous, Tanzanian lady presented with one-year history of abdominal swelling and secondary amenorrhea. Examination revealed a palpable, suprapubic mass, and a pelvic ultrasound showed features of ovarian tumour. An explorative laparotomy was performed to confirm the diagnosis. A bilateral salpingo-oophrectomy and hysterectomy was performed for huge bilateral ovarian tumours. Histopathological examination confirmed ovarian leiomyoma. Challenges remain in the management of bilateral primary ovarian tumours, especially for young and nulliparous women. Pre-laparotomy diagnostic measures are important so as to know the type of the tumour you are dealing with and plan the appropriate management.

Keywords: ovary, leiomyoma, tumours, laparotomy, Tanzania

\section{Introduction}

Primary leiomyoma of the ovary is rarely described in literature accounting for only 0.5 $1 \%$ of all benign ovarian tumours. Bilateral involvement affects women below 35 years of age (Usta et al., 2006; Wei et al., 2008; Tomas et al., 2009; Esch et al., 2011). Bilateral involvement is hard to find, only a few cases have been described in English journals (Esch et al. 2011) Most of the primary leiomyomas are small and asymptomatic, making them difficult to detect during physical examinations (Lim \& Jeon, 2004). Management of this condition is usually challenging, as the diagnosis is usually made by Histopathological examination (Lim \& Jeon, 2004; Esch et al., 2011). Most of the time, it can be confused with other benign tumours like fibroma/thecoma of which have similar gross and histologic presentation. We present a case of bilateral ovarian leiomyoma in a young, nulliparous woman.

\section{Case presentation}

A 29-year-old nulliparous, Tanzanian lady presented with abdominal swelling and amenorrhea for one-year prior. She reports to have noticed a slow growing abdominal mass that was not causing any pain or discomfort. The mass appears to be pronounced with full urinary bladder. Additionally, she presented with amenorrhea for the same duration as that of abdominal swelling. However, she denied using any form of contraception. Before the current problem, she reports to have had irregular menstrual pattern since menarche. Apart from irregularities, she also complains of prolongation of menstrual flow seven to ten days.

\footnotetext{
*Correspondence: Anthony Massinde; E-mail: amassinde@yahoo.com
} 
Upon examination, she was fairly stable with good nutritional status, not pale, not jaundiced, no palpable lymph nodes and no oedema. Her vital signs were as follows: blood pressure $=120 / 80 \mathrm{mmHg}$, pulse rate $=78$ beats $/$ minute, respiratory rate $=$ $22 \mathrm{cycle} /$ minute and temperature $=36.5^{\circ} \mathrm{C}$. Abdominal examination revealed distension of suprapubic area, some scar marks from traditional healer and abdomen moving with respiration. A mass was palpated on suprapubic area, which seemed to arise from pelvic region; it was mobile, non-tender, firm and a size equivalent to uterine fundus of 18 weeks. Kidneys, spleen and liver were not palpated. There was a dull percussion note at suprapubic area with normal note on other areas. There was no fluid thrilling, neither shifting dullness and the bowel sounds were normal. On vaginal examination, she had normal external genitalia, vaginal mucosa appeared to be smooth, cervix was healthy and its movement did not elicit tenderness. Although a mass was palpated, its relation to uterus or ovaries was not established. The impression at that moment was pedunculated uterine fibroids with differential diagnosis of ovarian tumour.

She was sent for trans-abdomino-pelvic ultrasound, which revealed a huge hyperechoic mass, measuring about $8.5 \mathrm{~cm} \times 6.6 \mathrm{~cm}$ located on left adnexae with clear margins. Right adnexae also had a solid mass measuring $11.4 \mathrm{~cm} \times 9.6 \mathrm{~cm}$. There was no ascites and no fluid in the Pouch of Douglas. The liver, spleen, and kidney, appeared to be normal, and there was no apparent lymphadenopathy. The conclusion was bilateral, huge, solid ovarian tumours. Her haemoglobin level was $13.2 \mathrm{~g} / \mathrm{dl}$, blood type $O$ positive and normal chest $x$-ray.

She was counselled and agreed to exploratory laparotomy. Intra-operatively, bilateral ovarian solid masses were found. Left tumour measured about $12 \mathrm{~cm}$ by $10 \mathrm{~cm}$ and the right one about $8 \mathrm{~cm}$ by $10 \mathrm{~cm}$. Both masses had irregular surface with some cystic areas. The ovarian tissue could not be identified easily. The uterus was found to be normal and the Pouch of Douglas was free of fluid and nodulation. No ascites or obvious lymph nodes were involved. Other visceral organs were normal.

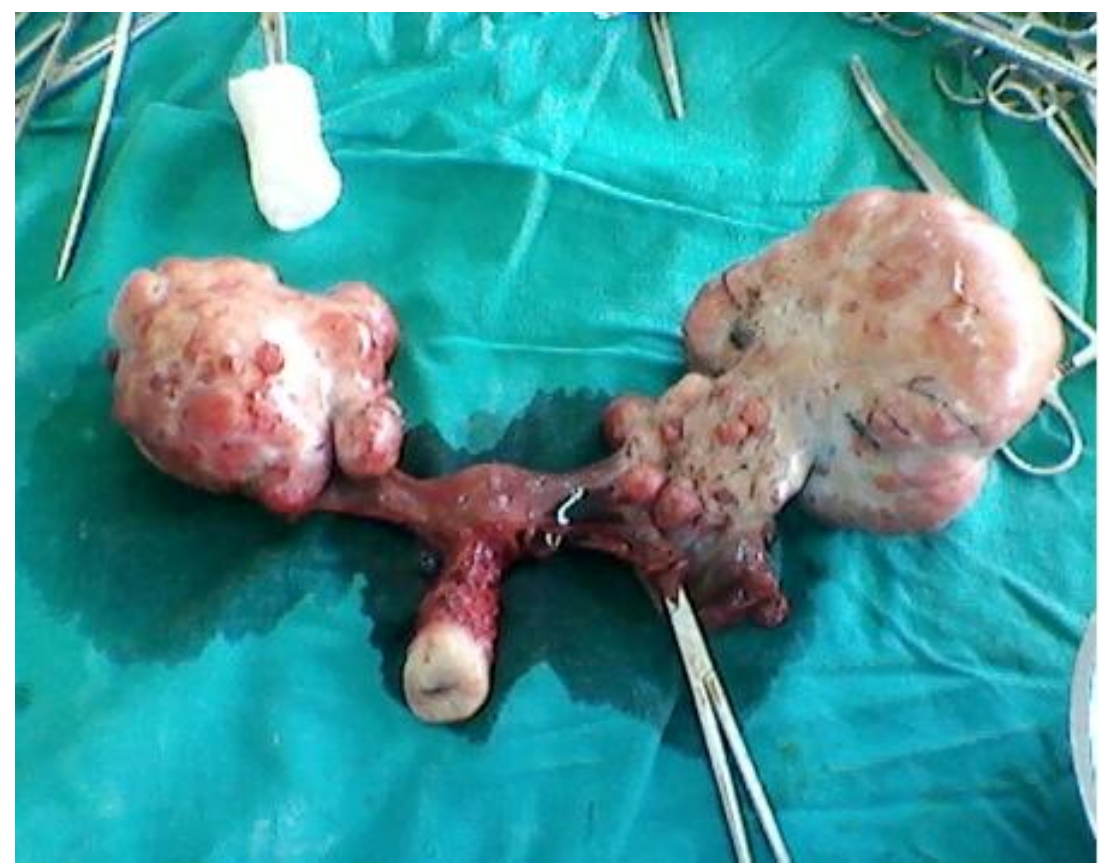

Figure 1: A gross specimen of a normal uterus with bilateral ovarian masses

Bilateral salpingo-oophrectomy and hysterectomy was performed and specimens were taken for histopathological examination. The gross pathology report revealed a small 
uterus of $7 \times 4 \times 2 \mathrm{~cm}$, attached with bilateral huge ovarian masses $12 \times 10 \times 6 \mathrm{~cm}$ (Figure 1). The cut surface revealed a solid whitish lesion with area of calcifications. Histological examination revealed the presence of elongated spindle-shaped cells of mesenchymal features with areas of whirling (Figure 2). All the cells had benign features though some areas show cellularity, consistent with Leiomyoma morphologically. Special stains were not available. Endometrium and cervix were normal.

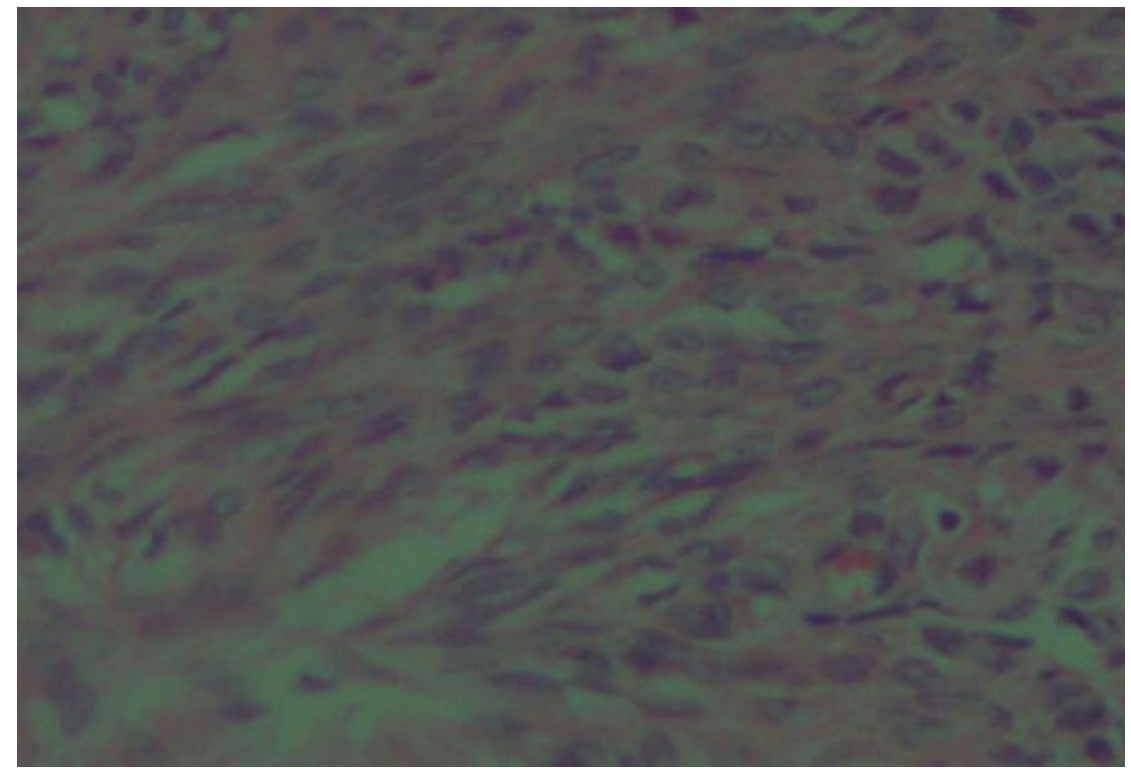

Figure 2: Histological section of the ovarian mass

She had uneventful postoperative period and discharged home on the third day postlaparotomy. A month later she was seen at outpatient clinic. She was doing well; her wound was completely healed. Hormonal replacement therapy was put into consideration. However, she did not show up in subsequent visits.

\section{Discussion}

Primary ovarian leiomyoma is one of the rare conditions to be described in the literature; it may be cystic or solid (Usta et al., 2006; Wei et al., 2008; Tomas et al., 2009; Esch et al., 20122; Choi et al., 2012). Since most of them are small and asymptomatic, they may be found incidentally during histological examination following removal of the reproductive organs (Usta et al. 2006; Tomas et al. 2009). It is said to originate from smooth muscle cells in the ovarian hilar blood vessels. Other possible origins include cells in the ovarian ligament, smooth muscle cells or multipotential cells in the ovarian stroma, undifferentiated germ cells or cortical smooth muscle metaplasia (Wei et al. 2008; Tomas et al. 2009).

Development of this ovarian leiomyoma is thought to mimic that of uterus. In this case, being nulliparous at the age of 29 years was likely to be an additional risk factor. Due to distortion that occurs in ovarian leiomyoma, it was difficult to identify normal tissue. In absence of frozen section, you would not be able to distinguish from malignant ovarian tumour. Diagnosis of this type of tumour is often difficult, and it is usually diagnosed through histopathology. However, in a well-resourced health facility, diagnostic laparoscopy and MRI/CT can sometimes be used to diagnose/differentiate it from leiomyosarcoma and plan for appropriate management (Tamada et al. 2006; Esch 
et al. 2011). Some cases have shown slight elevation of tumour markers such as CA 125 (Kandalaft \& Esteban, 1992; Marci \& Vasilev, 1993; Esch et al., 2011).

Its management may be difficult due to its rarity and lack of necessary diagnostic equipment. As for our case, partial oophrectomy could have been of advantage since she was nulliparous so as to improve fertility. Otherwise, ovarian preservation during ovarectomy may also allow the patient to later undergo in-vitro fertilization. This could be done through oocyte donation with the help of programmed hormonal supplement for endometrial and embryo development (Speroff \& Fritz, 2005). In a patient with completed family, bilateral oophrectomy and hysterectomy has been preferred as surgical treatment with excellent prognosis (Wei et al. 2008, Choi et al. 2012).

\section{Conclusion}

Bilateral primary ovarian leiomyoma still brings a challenge in its management, especially for young, nulliparous woman. Pre-laparotomy diagnostic measures are important so as to know the type of the tumour you are dealing with and plan the appropriate management.

\section{Acknowledgements}

We would to thank Liza Plafsky from Baylor Tanzania for her technical advice.

\section{Author's contributions}

All authors read and approved the final version of the manuscript.

\section{Conflict of Interest:}

The authors declare that they have no competing interests.

\section{References}

Choi, S.Y., Park J.S., Lee J.W., Kim B.G. \& Bae D.S. (2012) A rare case of primary ovarian leiomyoma with atypical meigs' syndrome. Korean Journal of Obstetrics and Gynecology 55, 285-289.

Esch, E.M.G.V., Wijngaarden, S.E.V., Schaafsma, H.E., Smeets, M.J.G.H. \& Rhemrev, J.P.T. (2011) The diagnostic and therapeutic approach of a primary bilateral leiomyoma of the ovaries: a case report and a literature review. Archive of Gynecology \& Obstetrics 283, 1369-1371.

Kandalaft, P.L. \& Esteban, J.M. (1992) Bilateral massive ovarian leiomyomata in a young woman: a case report with review of the literature. Modern Pathology 5, 586.

Lim, S.C. \& Jeon, H.J. (2004) Bilateral primary ovarian leiomyoma in a young woman: case report and literature review. Gynecologic Oncology 95, 733-735.

Macri, C.I. \& Vasilev, S.A. (1993) Bilateral giant primary ovarian leiomyoma. Journal of Reproductive Medicine 38, 480.

Speroff, L. \& Fritz, M.A. (2005) Assisted Reproductive Technologies. In Clinical Gynecologic Endocrinology and Infertility, eds. L. Speroff \& M. A. Fritz.

Tamada, T., Sone, T., Tanimoto, D., Higashi, H., Miyoshi, H., Egashira, N., Yamamoto, A. \& Imai, S. (2006) MRI appearance of primary giant ovarian leiomyoma in a hysterectomised woman. British Journal of Radiology, 79, e126 - e128. 
Tomas, D., Leniček, T., Tučkar, N., Puljiz, Z., Ledinsky, M. \& Krušlin, B. (2009) Primary ovarian leiomyoma associated with endometriotic cyst presenting with symptoms of acute appendicitis: a case report. Diagnostic Pathology 4, 1-4.

Usta, U., Karada, N., Turkmen, E., Haltafi, H. (2006) Primary leiomyoma of the ovary. Trakya Üniversitesi Tip Fakültesi Dergisi 23, 39-42.

Wei, C., Lilic, N., Shorter, N. \& Garrow, E. (2008) Primary ovarian leiomyoma: a rare cause of ovarian tumor in adolescence. Journal of Pediatric \& Adolescent Gynecology, 21. 